\title{
Evaluation of Prescribing Patterns Using WHO Indicators at Out Patient Department of A Private Hospital in Warangal
}

\author{
Durga.P, Abhinav.P, Varun Raj.K, Kishore.P \\ Department of Pharmacy Practice, Care College of Pharmacy, Oglapur, Damera, Warangal, Telangana, India
}

\begin{abstract}
A cross sectional study was conducted in Out Patient Department of Primary Care Hospital in Warangal. 502 prescriptions were randomly collected over a period of one month and recorded over a 'prescribing indicator form'. The data was analyzed using WHO indicators. Average number of drugs per encounter was 3.45. Encounter with an antibiotic prescribed was $46.21 \%$, with a FDC was $84.66 \%$, with an injection prescribed was $7.76 \%$. The most common group of drug prescribed was vitamins (19.1\%) followed by Antiulcer (12.34\%) Antimicrobials (11.78\%), Analgesics (10.22\%).The findings of this study revealed that drug utilization pattern was not optimal in accordance with the standard values of WHO prescribing patterns.
\end{abstract}

Keywords: Prescription pattern, Out patients, WHO indicators, Rational use of drugs, Essential Drug List

\section{Introduction}

The quality of prescribing medications is major determinant and plays crucial role in providing good health care and in the treatment of serious health conditions. The availability and affordability of good quality drugs and their rational use is needed for effective health care [1].In 1985, World Health Organisation (WHO) defined that 'Rational use of drugs requires that patients receive medications appropriate to their clinical needs, in doses that meet their own individual requirement for an adequate period of time and at the lowest cost to them and their community'. Common irrational uses of drugs include over or under use of medication, high cost of drugs, more usage of injections and antibiotics, poly pharmacy, violation of standard treatment guidelines or not from Essential Drug List ,usage of brands instead of generic names are the major problems of present- day medical practice. The consequences of these lead to ineffective treatment, development of resistance to antibiotics, adverse effects and economic burden on patients [2].

Out of the total health budget, one third of countries spent $30-40 \%$ on drugs, many of which prescribed irrationally. The GNP (gross national product) of these countries get almost doubled in every 16 years, however, their expenditure on drugs gets doubled in every 4 years [3]

Essential drugs offer a cost effective solution to many health problems in developing countries. The first model list of essential drug was published by WHO in 1977 and in 2002.WHO coined the term essential medicine as those medicine that satisfy the priority health care needs of the population. Essential medicines are not in access of $30 \%$ of population of World. In developing countries $60-80 \%$ of people do not have access to essential medicines this is due to low income and rest of the people have access to essential medicines receive wrong medication, inappropriate dosage[4]. Rational use of drugs is achieved if prescribers follow essential drug list and essential drugs are available on regular basis.

A study of prescription patterns is an important tool to determine rational drug therapy and maximize utilization of resources. To improve the overall drug use, especially in developing countries, international agencies like the World Health Organization (WHO) and the International Network for the rational use of drugs (INRUD) have applied themselves to evolve standard drug use indicators [5]. The components of core indicators are: prescribing indicator, patient indicator, and facility indicator. For the present study, we took the prescribing indicators which are as follows:

1. Average number of drugs per prescription

2. Percentage of drugs prescribed by generic names

3. Percentage of prescription with an injection prescribed

4. Percentage of prescription with an antibiotic prescribed

5 Percentage of drugs prescribed from essential drug list (EDL)

Studies in different countries evaluated the prescribing indicators in different health care settings. However the present study provides data which will be useful for comparison when in future any drug utilization study is carried out. The present study aimed at evaluation of prescribing indicators in the prescription of a private medical practitioner of primary health care centre in Warangal. 


\section{Methods}

Institutional ethics committee approval was obtained. A cross-sectional study was carried out for a period of one month in a private clinic providing the outpatient services with laboratory facilities and pharmacy. 502 prescriptions were randomly collected and were recorded over a WHO Prescribing Indicator Form. The data was analyzed to find out the prescribing pattern in the hospital using the WHO prescribing indicators.

WHO Prescribing Indicators [1]

1. Average number of drugs per encounter: Average, calculated by dividing the total number of different drug products prescribed, by the number of encounters surveyed. It is not relevant whether the patient actually received the drugs.

2. Percentage of drugs prescribed by generic name: Percentage, calculated by dividing the number of drugs prescribed by generic name, by the total number of drugs prescribed, multiplied by 100 .

3. Percentage of encounters with an antibiotic prescribed: Percentage, calculated by dividing the number of patient encounters during which an antibiotic is prescribed, by the total number of encounters surveyed, multiplied by 100 .

4. Percentage of encounters with an injection prescribed: Percentage, calculated by dividing the number of patient encounters during which an injection is prescribed, by the total number of encounters surveyed, multiplied by 100 .

5. Percentage of drugs prescribed from essential drugs list or formulary: Percentage, calculated by dividing the number of products prescribed which are listed on the essential drugs list or local formulary (or which are equivalent to drugs on the list), by the total number of products prescribed, multiplied by 100

\section{Results}

A total of 502 prescriptions were randomly collected and analyzed. A total of 1735 drugs were prescribed [Table 1] Average number of drugs per encounter was 3.45. Drugs prescribed from essential drugs list (India) were 496 (28.58\%).Drugs prescribed from essential drugs list(WHO)were 423(24.3\%). Total number of prescriptions with an antibiotic was $232(46.21 \%)$. Total number of prescriptions with an injection was $39(7.76 \%)$ Total number of prescriptions with a FDC was $425(84.66 \%)$.

Table 1: prescribing Indicators

Prescribing Indicators No. $(\%)$

Total number of prescriptions analyzed

502

Total number of drugs prescribed

Average number of drugs per encounter*

3.45

Drugs prescribed by generic name*

$1696(97.7 \%)$

Drugs prescribed from essential drugs list (India)*

$496(28.58)$

Drugs prescribed from essential drugs list (WHO)*

423(24.3)

Total number of prescriptions with an antibiotic*

232(46.21)

Total number of prescriptions with an injection* 39(7.76)

Total number of prescriptions with a FDC

*WHO prescribing indicators

The most common group of drugs prescribed was Multivitamins(19.1\%),Antiulcer(14.1\%) followedbyAntimicrobials(13.3\%)Analgesics(11.7\%),Antidiabetic(4.4\%),Antihypertensives(9.3\%), Antihyperlip edeimc $(1.5 \%)$, Anticonvulsants(5.4\%), Antidepressants(5.1\%).

TABLE 2: Distribution of drugs according to the disease condition

\begin{tabular}{|l|l|l|}
\hline S.No & Disease & Number of drugs \\
\hline 1 & Antiulcer & 245 \\
\hline 2 & Antimicrobials & 232 \\
\hline 3 & Analgesics & 203 \\
\hline 4 & Antidiabetic & 77 \\
\hline 5 & Antihypertensives & 162 \\
\hline 6 & Antihyperlipidemic & 27 \\
\hline 7 & Anticonvulsants & 60 \\
\hline 8 & Antidepressants & 90 \\
\hline 9 & MVT & 332 \\
\hline 10 & Others & 307 \\
\hline Total & & 1735 \\
\hline
\end{tabular}


GRAPH 1: Commonly prescribed drug categories:

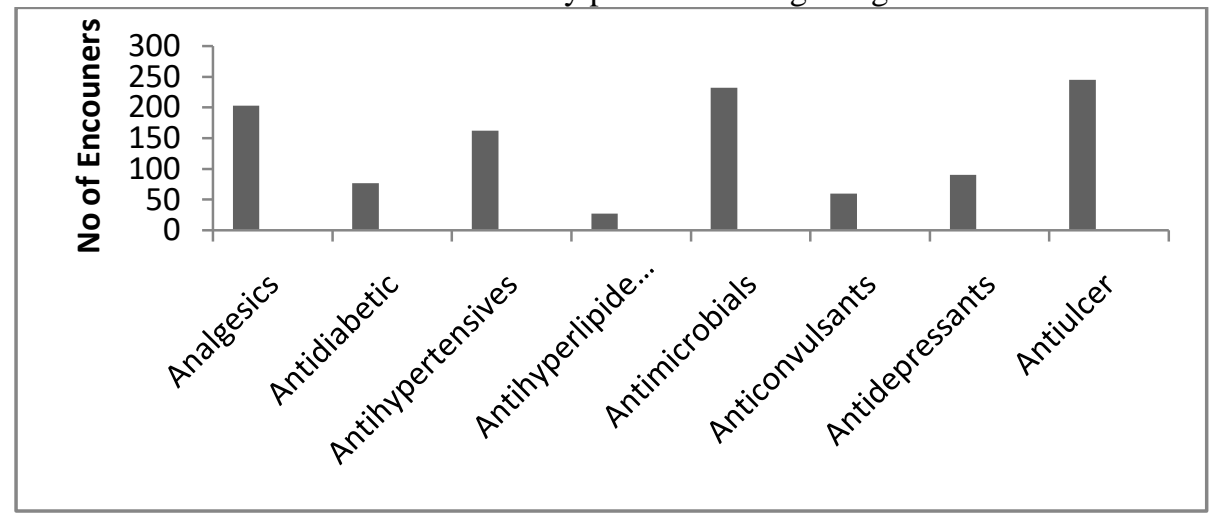

Among prescribed antimicrobials, Fluroquinolones (51.2\%) are more followed by cephalosporins(18.5\%), Tetracyclines $(2.58 \%)$ and Others(28.4\%)..

GRAPH 2: Commonly prescribed antibiotics

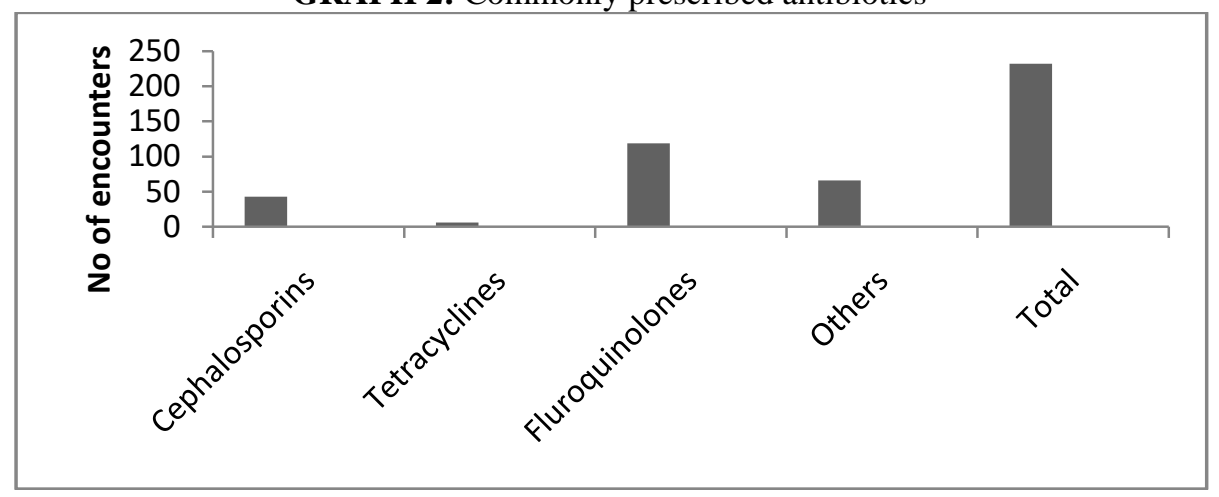

The most common group of dosage forms prescribed other than tablets were capsules(42.9\%), Syrups(30.6\%), Gels $(13.68 \%)$ and injections $(12.7 \%)$.

GRAPH 3 : Commonly prescribed dosage forms:

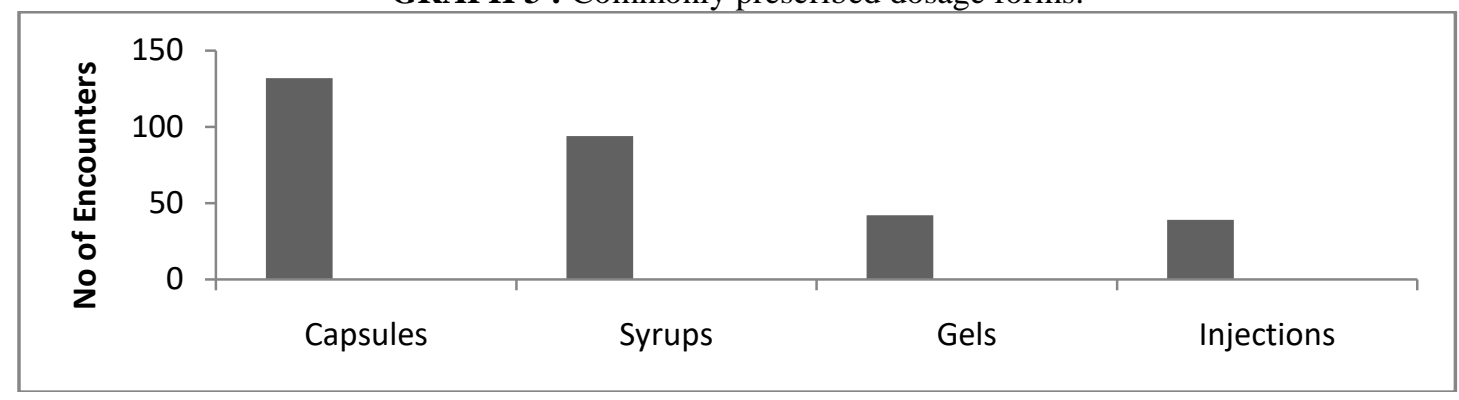

\section{Discussion}

The average number of drugs prescribed per encounter was 3.45.It was less than that reported in most of the government setups across Indian cities. The more closet being Allahabad (3.52)[6] . It was more than that reported compared with Nagpur (3.40) [7] and Delhi (3.03)[8]. International studies report values ranging from 1.3 in Zimbabwe [9] to 4.51 in Pakistan.[10] As per WHO ,the average number of drugs per prescription should be 1.6-1.8. Our study reveals poly pharmacy which reflects the usual practice of private set up clinics.

The most common drug prescribed was vitamins (19.1\%) followed by Antiulcer (12.34\%) Antimicrobials (11.78\%),Analgesics(10.22\%). This shows that prescribers are more intended to prescribe vitamins , ulcer healing drugs, antimicrobials and analgesics commonly.

In this study, the percentage of prescriptions with antibiotics was $46.21 \%$. According to WHO $15-25 \%$ of prescriptions with antibiotics is expected in most of the developing countries where infectious diseases are more prevalent [11]. Studies from other states (Tamil Nadu(55\%), Madhya Pradesh(60.9\%) which are highly reported 
and New Delhi(29.9\%), Uttar Pradesh(39.9\%) of India are reported which are less compared to this study of prescriptions where antibiotics were prescribed[12] This phenomenon is very high in some of the developing countries like Pakistan (78\%) [10], eastern Nepal (79.9\%)[13]. Various studies from India also report a high rate ranging from $40-80 \%[14]$.

The most common antibiotic category prescribed was Fluroquinolones( $51.2 \%)$, followed by cephalosporins(18.5\%), Tetracyclines $(2.58 \%)$ and others $(28.4 \%)$. The prescribers need to be extra cautious before prescribing any antibiotic to avoid unnecessary burden on patient and development of resistance.

The use of injection for treatment is accompanied with a variety of disadvantages including sepsis at administration, increased risk of tissue toxicity from local irritation, costly, difficulties in correcting the error, thus, WHO recommended that less than $10 \%$ prescription should include one or more injections.[15], but the percentage observed in the present study was $7.76 \%$ in the total sample.

Essential drug offers a cost-effective solution to many health problems in a developing country. They should be selected with due regard to disease prevalence, be affordable, with assured quality and be available in the appropriate dosage forms. In our study, the percentage of drugs prescribed from the essential drugs list of India was $28.5 \%$ which was low as compared to that reported by study conducted in pune [1] and essential drug list of WHO was only 24.3\%.This may be due to lack of awareness of Essential Drug List.

\section{Conclusion}

The prescribing practice in this study was not satisfactory, as findings of this study revealed that drug utilization pattern was not optimal in accordance with the standard values of WHO prescribing patterns. The drug prescribing practices should be improved regardless of the level of health care delivery. The complete outcome of the prescribing desire will be successful only when the patient receives rational treatment for particular disease. The formularies in hospital especially of primary health care hospital, the prescribing should be based on the essential drugs and the prescribers should be encouraged to prescribe as per necessary and eliminate the unnecessary drugs which significantly lead to rational drug therapy. This study will act as feed back to the prescribers so as to create awareness about the rational use.

\section{References}

[1]. Bhavesh k. Lalan, r. S. Hiray, b. B. Ghongane: Drug prescribing pattern of out patient department ina tertiary care hospital in maharastra. International journal of Pharma and Bio sciences,3(3), 2012,225-229.

[2]. Tamesgen Sidamoro,Kassa Daka Gidebo,Zewde Zemma kanche ,Eskinder Wolka Woticha: Evaluation trends of drug prescribing patterns based on WHO prescribing indicators at outpatient departments of four gospitals in south Ethiopia , Drug Design ,Development and Therapy,9,2015,4551-4557

[3]. Ashley G.Saures, Mahikumar P.Board.,Sheena Thomas : Analysis of Out Patient Prescription Pattern by MBBS and MD Doctors in Ahmedabad City, World Journal Of Pharmaceutical Sciences,5(1),2015,1246-1255.

[4]. WHO,Promoting rational use of medicine: core components, WHO policy perspectives on Medicines, Geneva September 2002, accessed 28 June $2012<$ http://apps.who.int/medicinedocs/en/d/Jh3011e/>

[5]. World Health Organisation.How to investigate Drug use in Health Facilities.Selected Drug use Indicators.WHO/DAP/93.1 Geneva: World Health Organisation:1993a.Available at http://www.apps.who.int/medicinedocs/pdf/s2289e.pdf. Accessed on26 May 2015.

[6]. K.U.Ansari, S.Singh, R.C.Pandey, Evaluation of prescribing patterns of doctors for rational therapy,Indian journal of Pharmacology,30,1998,43-46.

[7]. Thawani VR,Motghare VM,purwar MB,Pagare A,Drug utilization studies in inddor ANC patients of Govt,College Hospital,Nagapur.Journal of Academy of Hospital Administration,9,1997,1408-1410.

[8]. Biswas NR,Jindal S,Siddiquei MM et al Patterns of prescription and drug use in opthamology in a tertiary hospital in Delhi,Br J Cli Pharmacol ,51,2001,267-269.

[9]. Hogerzeil HV, Bimo, Ross-Degnan D,Laing RO et al, Field tests for rational drug use in twelve developing countries. Lancet,342,1993,1408-1410.

[10]. Das N, Khan AN, Badini ZA, Baloch H,Prescribing practices of consultants at Karachi, Pakistan. J Pak Med Assoc, 51,2001,:74-77.

[11]. World Health Organization. How to investigate drug use in health facilities. Selected drug use indicators.WHO/DAP/93.1. WHO, Geneva, 1993.

[12]. Pragnadyuti Mandal, Shirsendu Mondal, Anjan Das, Musfikur Rahaman, Manab Nandy, Swapan Jana, Evaluation of prescribing indicators in prescriptions of private practitioners in Kolkata, India,_International Journal of Basic \& Clinical Pharmacology 4 (5) ,2015,919.

[13]. Rauniar GP, Shahanas MS, Dos BP, Naga Rani MA, A prospective study of dental pattern and drug utilization at the dental department of a tertiary care teaching hospital in eastern Nepal. Journal of Nepal Medical Association, 40,2001, 6-11.

[14]. Kumari Indira KS, SJ Chandy, L ,Jeyaseelan, Rashmi Kumar, Saradha Suresh, Antimicrobial prescription patterns for common cute infections in some rural \& urban health facilities of India. Indian J Med Resm, 128,2008,165-171.

[15]. Panda J et al Evaluation of the rationality of some FDCs: Focus on anti hypertensive drugs. Indian J Pharm Sci.,68,649-53. 